\title{
Remote Associates Test and Alpha Brain Waves
}

\author{
Henk J. Haarmann, Timothy George, Alexei Smaliy, Joseph Dien'
}

\begin{abstract}
:
Previous studies found that performance on the remote associates test (RAT) improves after a period of incubation and that increased alpha brain waves over the right posterior brain predict the emergence of RAT insight solutions. We report an experiment that tested whether increased alpha brain waves during incubation improve RAT performance. Participants received two blocks of RAT items (RAT1 and RAT2), with the second block consisting of items that were not solved during the first block. Participants were randomly assigned to three groups, which were matched for their number of RAT1 solutions. Participants in an alpha-up neurofeedback group aimed to increase their alpha brain waves over the right posterior brain in between the two blocks, whereas participants in an alpha-down neurofeedback group aimed to decrease these same brain waves. A third group of participants did not perform neurofeedback and proceeded immediately from the first to the second block of RAT items. We found evidence for more RAT2 solutions in participants who interrupted their RAT performance with neurofeedback, especially in ones who showed high alpha brain waves during neurofeedback. These results are consistent with the notion that an alert but relaxed mental state, indexed by alpha brain waves, may aid the read out of an implicitly activated memory network of weak associates.
\end{abstract}

Keywords:

Remote Associates Test, Alpha brain waves, EEG neurofeedback, problem incubation

${ }^{1}$ University of Maryland

The Journal of Problem Solving • volume 4, no. 2 (Spring 2012) 


\section{Introduction}

Problem solving often involves arriving upon a solution after activating a network of mutually supporting thought associations. However, when nonsolutions are highly activated in memory because they are strongly associated with the terms of the problem statement, they may hinder retrieval of a nonobvious solution. Incubating on a problem-that is, putting it aside for while-may increase one's chances of finding a solution in such a case because it allows one to overcome mental fixation on dominant but irrelevant associations that do not lead to the solution.

This analysis raises the question of what type of thinking mode and associated brain state promote an incubation effect for such a problem. In this study, we addressed this question in the context of the remote associates test (RAT), inspired by a report of an association between alpha brain waves and RAT insight solutions (Jung-Beeman, et al., 2004) and the possibility that these brain waves might provide the problem solver with easier access to weak but relevant associations. We predicted that individuals with high alpha brainwaves during incubation produce more RAT solutions. EEG (Electro-encephalography) neurofeedback training, aimed at increasing versus decreasing alpha brainwaves, was provided during incubation to examine the causal relation between these brainwaves and RAT performance. To enhance the chances of finding an incubation effect on RAT performance, we induced mental fixation using a method introduced by Smith and his colleagues (Smith \& Blankenship, 1991). The rationale for this approach was motivated as follows.

Mednick and Mednick suggested that retrieving weak but relevant associations is a hallmark of cognitive creativity (Mednick, 1962) and created the RAT as a task paradigm for investigating this process (Mednick, 1968; Mednick \& Mednick, 1967). On each trial in this test, participants are presented with three unrelated words and have to produce an answer word that connects all three cues by forming a familiar phrase with each of them. For example, the word cheese connects the three cue words blue, knife, cottage, by forming the phrases blue cheese, cheese knife, and cottage cheese. Thinking of the related word sky while looking at this problem makes it more difficult to find the answer cheese. As this example illustrates, good performance on the RAT requires countering mental fixation on strong but misleading meaning associations and broadening the search of semantic memory so that relevant weaker meaning associations, such as blue cheese, are considered as well.

RAT solutions are even more difficult to find when mental fixation is experimentally induced through a changed test context in which strong, misleading semantic associates of the cue words are read immediately before the RAT problem, or presented together with it (Kohn \& Smith, 2009; Smith \& Blankenship, 1991; Wiley, 1998). For example, the answer to the problem lick, sprinkle, and mines is salt. This answer is more difficult to find when

- volume 4, no. 2 (Spring 2012) 
a related word is presented in parentheses after each cue word, as in as in lick (tongue), sprinkle (rain), and mines (gold) (Kohn \& Smith, 2009; Smith \& Blankenship, 1991).

The negative impact of this kind of experimentally induced mental fixation on RAT performance can be reduced by incubation (Kohn \& Smith, 2009; Smith \& Blankenship, 1991; Wiley, 1998). In the prototypical incubation paradigm, a problem solver who fails to solve a problem during a first work period receives another attempt to solve the problem during a second work period. The two work periods occur either in immediate succession without interruption (nonincubation, control condition) or they are separated by an intervening period during which the problem solver's attention is distracted away from the problem (incubation condition), by asking the problem solver to perform a different task, such as studying a story (Smith \& Blankenship, 1991), monitoring digits (Kohn \& Smith, 2009), solving anagrams (Wiley, 1998), or relaxing (Browne \& Cruse, 1988). In both the incubation and control condition, one expects an added benefit from the second work period in terms of increasing the likelihood of solving the problem. When this benefit is greater in the incubation than control condition, there is evidence of an incubation effect (Dorfman, Kihlstrom, \& Shames, 1996; Smith \& Blankenship, 1989). Incubation effects can be due to various potential mechanisms, such as fixation forgetting, recovery from fatigue, unconscious activation, intermittent conscious problem solving, and encountering problem solving clues. Which mechanisms are necessary and sufficient for incubation to occur is a matter of ongoing study and debate (for a review of theories see Helie \& Sun, 2010).

Across several experiments, Smith and colleagues found evidence of an incubation effect on RAT performance, when during the first work period mental fixation had been experimentally induced by presenting strong, misleading associates of the cue words immediately before or together with the problem. These effects occurred regardless of whether the incubation period followed each single RAT problem or a list of (ten to twenty) RAT problems and with incubation periods lasting from forty seconds to five minutes (Kohn \& Smith, 2009; Smith \& Blankenship, 1991). From the finding that the incubation effect occurred even when the distracters were presented before instead of together with the RAT problem, Smith and Blankenship inferred that the mental fixation was mediated by associative processes in memory and not merely perceptual in nature. They concluded that incubation under these conditions facilitated forgetting of the misleading cues arising from mental fixation. Evidence for the fixation forgetting explanation of incubation effects has also been obtained in studies using rebus puzzles (Smith \& Blankenship, 1989) and anagrams (Vul \& Pashler, 2007) as the problem solving task. However, these findings do not exclude the possibility that incubation effects may be due to contributions from other psychological mechanisms, such as unconscious activation in implicit memory taking place during the incubation period (Dorfman, et al., 1996). Moreover, RAT performance does not always benefit from incubation. Negative incubation effects (i.e., a greater advantage of retesting in the nonincubation than incubation condition) have been obtained under 
conditions in which mental fixation is induced by presenting RAT problems that have been specifically constructed to activate strong, misleading associates based on the problem solver's prior domain-specific knowledge (Wiley, 1998).

The idea that RAT problems are difficult to solve due to having to overcome competition from misdirected associations has also been central in studies by Beeman, Bowden, and their colleagues on the neurobiological mechanisms mediating RAT problem solving (Bowden \& Beeman, 1998, 2000, 2003a, 2003b; Jung-Beeman, et al., 2004). Across several of their experiments, participants tried to solve compound remote associate (CRA) problems for periods varying between 1, 2, 7 to 15 seconds after which they were presented with the answer or an unrelated word in either the left or right visual field (LVF or RVF) for a naming response or solution decision (Bowden \& Beeman, 1998, 2000). CRA problems are a type of RAT problem in which the answer word forms a single compound word or common word pair with each of the three cue words (e.g., schoolhouse, high school, school district), reducing the total semantic space that needs to be searched. Priming effects for answer words were obtained for problem periods of up to 15 seconds with LVF/Right hemisphere $(\mathrm{RH})$ presentation but only up to 2 seconds with RVF/Left hemisphere (LH) presentation. Bowden and Beeman interpreted these findings as suggesting that solutionrelated activation persists in the $\mathrm{RH}$ longer than in the $\mathrm{LH}$. They furthermore suggested that this activation might be unconscious due to a more diffuse representation of meaning in the RH (Jung-Beeman, 2005), which may be overshadowed by more focal misdirected representations in the $\mathrm{LH}$.

Jung-Beeman et al. (2004) used EEG to record the neural-electrical activity of the brain while participants attempted to solve CRA problems. Immediately after producing the answer to a problem, participants were asked to indicate whether they achieved the response with or without insight. Insight was defined prior to the experiment as the subjective awareness of an "Aha" experience, in which the solution appears to come to one's mind suddenly and it is immediately obvious that is the correct the solution. A half second before the response, there was a sudden burst in the EEG spectral power within the gamma frequency band $(39 \mathrm{~Hz})$ over the right temporal lobe for insight problems relative to noninsight problems, which appeared to reflect the sudden conscious awareness of the RAT solution (Jung-Beeman, et al., 2004). This insight-related burst in gamma band power was preceded by an insight-related burst in EEG spectral power in the alpha band $(8$ to $12 \mathrm{~Hz}$ ) over right parietal-occipital electrode PO8 that occurred in the 1.4 to 0.4 seconds before the response. Jung-Beeman, et al., suggested that the transient increase in alpha brain waves may have promoted "weaker processing about more distant associations between the problem words and potential solutions to gain strength, by attenuating bottom-up activation or other neural activity not related to solution that would decrease the signal-to- noise ratio for the actual solution" (p. 507). In other words, alpha brain waves may be hypothesized to reduce the introduction of task-irrelevant external and internal

- volume 4, no. 2 (Spring 2012) 
distracters into the mental workspace, thereby enabling better access to already primed representations, including weak associates.

Several other findings involving alpha brain waves are consistent with this hypothesis. Alpha brain waves are regular sinusoidal shaped brain waves oscillating at 8 to $12 \mathrm{~Hz}$, modulated by the thalamus and found in a distributed network of cortical areas affecting sensory, motor, and cognitive processes (Sabate, Llanos, Enriquez, Gonzalez, \& Rodriguez, 2011). It is more difficult to identify stimuli during visual perception when the overall power of alpha brain waves is high (Thut, Nietzel, Brandt, \& Pascual-Leone, 2006). Thus, the likelihood that such stimuli are identified decreases with increased occipital alpha brain waves. Through such a mechanism, increased alpha brain wave activity reduces the bottom-up activation of task-irrelevant and potentially distracting information. High pretask alpha brain waves immediately prior to the presentation of a stimulus are associated with a high reduction in alpha brain waves during the presentation of the stimulus (i.e., alpha de-synchronization) and good performance on cognitive tasks that require processing of that stimulus (e.g., memory recognition) (Klimesch, 1999). This performance advantage may be due to the reduced processing of internal and external distracters during the prestimulus period. When a person closes their eyes while in an alert and calm state, increased episodes of alpha brain waves can be observed in the raw EEG. In this state, external distracters are reduced due to eye closure and internal distracters may be reduced since a calm state is less likely to involve introduction of new and potentially misleading ideas through effortful processing. Alpha brain waves have been found to be higher during internally directed attention to imagined stimuli compared to externally directed attention to sensory stimuli (Cooper, Croft, Dominey, Burgess, \& Gruzelier, 2003) and also during short-term retention (Haarmann \& Cameron, 2005; Jensen, Gelfand, Kounios, \& Lisman, 2002), especially with increased processing load. These findings suggest that alpha brain waves help to maintain task-relevant representations in an activated state in memory, possibly by protecting them against the negative impact of distracters, for example, by inhibiting brain regions that would otherwise process these distracters (Jensen, et al., 2002; Klimesch, Doppelmayr, Rohm, Pollhuber, \& Stadler, 2000). Such a mechanism might also explain the finding of a positive association between alpha brain waves and verbal creativity (Fink, Grabner, Benedek, \& Neubauer, 2006; Fink \& Neubauer, 2006; Jausovec, 2000; Martindale \& Hasenfus, 1978; Martindale \& Hines, 1975). However, not all EEG studies find such an association (Danko, Shemyakina, Nagornova, \& Starchenko, 2009; Molle, Marshall, Wolf, Fehm, \& Born, 1999; Shemyakina \& Danko, 2007) and the conditions under which it appears remain to be elucidated (Dietrich \& Kanso, 2010).

If alpha brain waves reduce the influence of external and internal distracters, then increased alpha brain waves might promote activation of weak associates during incubation and thus RAT performance. The proposal that alpha brain waves, which reflect a 
calm and alert mental state, might benefit sensitivity to task-relevant associations during incubation is compatible with a recent theory of incubation and insight problem solving, the explicit-implicit interaction theory (Helie \& Sun, 2010). According to this theory, incubation enables an implicit memory system to contribute to problem solving free of the top-down bias of an explicit, rule-based memory system involved in effortful processing.

In the present study, we used EEG neurofeedback (NFB) during incubation in an attempt to examine the causal role of alpha brain waves on RAT performance, with participants in one group receiving NFB to increase alpha brain waves and participants in another group receiving NFB to decrease alpha brain waves. EEG NFB measures a person's ongoing neural electrical activity in a specific frequency band from the scalp-recorded electro-encephalogram (EEG), associates that activity online with visual or auditory information, and presents it as immediate feedback to allow a person to learn to increase or decrease the degree of EEG spectral power in that band (e.g., in the form of tone whose pitch represents the amount of alpha activity). Since the feedback can be viewed as a reward that shapes brainwave responses, EEG NFB has been described as a form of operant conditioning of brain waves (Sterman \& Egner, 2006). EEG NFB can change brain activity and improve cognitive performance in both clinical and nonclinical populations (Vernon, 2005; Vernon, et al., 2003), often after many training sessions (Leins, et al., 2007) but sometimes in as little as a single training session (Hanslmayr, Sauseng, Doppelmayr, Schabus, \& Klimesch, 2005). EEG NFB has been used to test hypotheses that associate changes in EEG spectral power with improvements in particular cognitive functions (for a review see Vernon, 2005), including attention and semantic working memory (Vernon, et al., 2003) and mental rotation (Hanslmayr, et al., 2005). The latter but not former study used alpha NFB. Hanslmayr and colleagues found that individuals who increased alpha power at right parieto-occipital sites prior to a mental rotation task improved their performance on that task.

In the present study, we used EEG NFB for the first time during problem incubation. To maximize chances of finding an effect of incubation against a no-incubation control condition, we presented each of the three cue words of a RAT problem with misleading distracters to increase mental fixation in view of the finding that RAT performance benefits from incubation under those conditions (Kohn \& Smith, 2009; Smith \& Blankenship, 1991). We adopted a NFB protocol for increasing alpha activity in a single 30-minute session (Fell, et al., 2002) and changed it in three ways. First, instead of basing the neurofeedback signal on an electrode over the center of scalp (i.e., Cz), we based it on an electrode over the right posterior occipital scalp. We specifically chose electrode PO8 for this purpose since it was the electrode location at which Jung-Beeman, et al. (2004) found increased alpha power prior to RAT insight solutions. Second, instead of presenting auditory feedback, we presented visual feedback, consistent with Jung-Beeman, et al.'s suggestion that the increased

- volume 4, no. 2 (Spring 2012) 
alpha power they obtained over PO8 could reflect inhibition of visual distraction, which in turn might have increased sensitivity to weak associates in their study. To elaborate on this point, the processing of visual target stimuli de-synchronizes alpha power (i.e., reduces alpha power) and this is also the case for the processing of continuously changing visual feedback information. We therefore reasoned that participants who attempt to increase their alpha power with such visual feedback might have to adopt a visual processing strategy that results in inhibition of the visual information from the feedback signal and thus a neurophysiological state that could be similar to the one that Jung-Beeman and colleagues hypothesize to increase sensitivity to weak associates. Indeed, in line with such a processing strategy, a post-experimental survey administered after pilot testing with our alpha NFB protocol, indicated that a majority of trainees reported either blurring their view of the visual feedback signal or maintaining it in their peripheral vision as a strategy to increase alpha power. Third, we included not only a NFB training condition aimed at increasing alpha brain waves, but also one for decreasing alpha brain waves. In view of the above considerations, we expected to find an incubation effect on RAT performance, especially in individuals with higher alpha brain waves during incubation as a function of individual differences and NFB condition.

\section{Experimental Methods}

\section{Participants and Design}

Seventy six University of Maryland students participated in the study. They were paid for their participation. Their average age was 20.1 years and 41 were female and 35 male. Participants were randomly assigned to one of three conditions: control $(N=25)$, alpha-up $(N=26)$, and alpha-down $(N=25)$. All participants completed a first block of RAT items (RAT1) and a second block of RAT items (RAT2) in which they attempted to solve previously unsolved RAT1 items. RAT1 and RAT 2 occurred in immediate succession (i.e., without interruption) in the control condition, whereas they were separated by an intervening incubation period of 35 minutes in the alpha-up and alpha-down conditions. During this period, participants used EEG neurofeedback (NFB) to increase alpha brain waves in the alpha-up condition and to decrease these waves in the alpha-down condition. The study was undertaken with the understanding and consent of each participant under a human subjects research protocol approved by the institutional review board of the University of Maryland.

\section{Materials}

Materials for the Remote Associates Test consisted of 124 CRA-type RAT problems drawn from the norms of Bowden and Beeman (2003), four of which were used for practice trials. 
For each of the three words in a RAT problem, the authors created a dominant associate word for display in parentheses adjacent to the problem word, for example, FRENCH (fry) SHOE (sock) CAR (garage). The materials are shown in Appendix A.

\section{RAT procedure}

RAT presentation was computer controlled. All participants received two blocks of RAT trials (i.e., RAT1 and RAT2). The order of trials within a block was randomized per participant. The two RAT blocks occurred in immediate succession without interruption in the control condition, whereas they were separated by approximately 30 minutes of NFB in the alphaup and alpha-down conditions. RAT1 consisted of 120 trials with one RAT item per trial. A participant's RAT2 items consisted of items that that particular participant had failed to solve during RAT1. Immediately after the end of RAT1, all participants were made aware that they would be given RAT2 in the same session. The sequence of events on a RAT1 trial was as follows. Participants were first presented with a "Ready?" prompt, which indicated that they should press the spacebar when they were ready for the problem. After an interval of $500 \mathrm{~ms}$, the RAT problem was displayed on a single line in the middle of the computer screen. Participants were instructed to press the space bar when they thought they knew the answer, type it in, and then press enter to proceed to the next trial. Participants had 15 seconds to think of an answer and press the space bar. If they did so, they were given an additional 10 seconds to type the answer. The RAT problem remained on the screen for a maximum duration of 15 seconds and disappeared when subjects finished typing their answer before this time had lapsed. The sequence of events on a RAT2 trial was the same as on a RAT1 trial with one exception: after trying to solve the problem, participants were furthermore asked to indicate whether they solved the problem with or without insight, by pressing "1" or "2" on the keyboard (counter-balanced across participants), after which they proceeded to the next trial. Participants were provided the following definition of an insight solution once at the start of the RAT2 block: "A feeling of insight is a kind of 'Aha!' experience characterized by suddenness and obviousness. You may not be sure how you came up with the answer, but are relatively confident that it is correct without having to mentally check it. It is as though the answer came into mind all at once-when you first thought of the word, you simply knew it was the answer. This feeling does not have to be overwhelming, but should resemble what was just described." Participants were instructed to report solution experiences that did not meet this definition as noninsight solutions.

\section{EEG neurofeedback procedure}

The Nexus-32 system (Mind Media, Ltd; Netherlands) was used for EEG NFB training and EEG recording. Participants were fitted with a stretch-lycra cap with openings for inserting $\mathrm{Ag} / \mathrm{AgCl}$ electrodes and injecting conducted gel. Scalp EEG was recorded from 22 channels

- volume 4, no. 2 (Spring 2012) 
arranged according to an extended International 10-20 system (FP1, FP2, F7, F3, Fz, F4, $\mathrm{F} 8, \mathrm{~T} 3, \mathrm{C} 3, \mathrm{Cz}, \mathrm{C4}, \mathrm{T} 4, \mathrm{~T} 5, \mathrm{P3}, \mathrm{Pz}, \mathrm{P} 4, \mathrm{~T} 6, \mathrm{O} 1, \mathrm{O} 2, \mathrm{PO} 7, \mathrm{PO}$, and outer canthus of right eye) in addition to left and right mastoids ( $A 1$ and $A 2$ ). The letters $F, C, T, P, O$, and PO indicate electrodes (channels) placed over the frontal, central, temporal, parietal, occipital, and parietal-occipital lobe, respectively, while odd and even numbers indicate electrodes placed over the left and right hemisphere, respectively. Electrode impedances were kept below 10K Ohms. The quality of the electrode connective to the skin was also checked with the Nexus-32 DC offset checking. Participants in all three conditions (alpha-up, alphadown, no-incubation control) were fitted with electrodes at the start of the experiment and the EEG was recorded during RAT1 and RAT2 and, in the alpha-up and alpha-down condition, also during the incubation period separating RAT1 and RAT2. The signals were sampled by a 24-Bit analog-digital converter at a sampling rate of $256 \mathrm{~Hz}$, bandpass filtered at $0.15-64 \mathrm{~Hz}$. On-line, digital bandpass, infinite impulse response (IIR) filters (Butterworth cascaded 3rd order) were used to determine peak-to-peak amplitudes (magnitudes) in each of the following frequency bands: delta $(1-4 \mathrm{~Hz})$, theta $(4-8 \mathrm{~Hz})$, alpha $(8-12 \mathrm{~Hz})$, beta $1(12-16 \mathrm{~Hz})$, beta2 $(16-20 \mathrm{~Hz})$, beta3 $(20-28 \mathrm{~Hz})$ (Fell, et al., 2002) and EMG (43-64 Hz). Participants were seated in a comfortable chair, made aware of the undesirable impact of movement and muscle tension on EEG recording (i.e. by looking at the effect of jaw clenching on their raw EEG), and instructed to limit eye blinking, any eye, tongue or jaw movement, clenching of the jaw, furrowing of the brows and forehead, and movement of the neck, upper torso, and fingers. They were asked to sit as still as possible and keep their eyes focused on the screen in front of them.

We adopted the following aspects of the alpha NFB protocol of Fell, et al. (2002). The NFB consisted of four baseline segments (B1, B2, B3, and B4) and nine training segments (T1 to T9), which were administered in the following order: B1 T1 T2 T3 B2 T4 T5 T6 B3 T7 T8 T9 B4. A single baseline and training segment lasted 1.5 and 2.5 minutes respectively, and a 20 -second rest separated adjacent training segments. EEG was recorded during baseline and training segments, but not during rest. The beginning and end of each of the baseline, training, and rest segments were indicated by a 3-second visual prompt (e.g., begin training; end baseline). The total duration of the NFB run, including baseline segments, training segments, and rests, was 30 minutes. Our alpha NFB protocol differed from the protocol by Fell et al. in several respects, as already described in the introduction.

During the NFB period in between RAT1 and RAT2, participants in the alpha-up and alpha-down condition watched a moving green bar with a line marking their individual alpha threshold, displayed at the center of the display screen. The height of the green bar changed in proportion to a trainee's alpha magnitude (smoothed by averaging across a running window of 10 samples), such that a higher bar corresponded to greater alpha in the alpha-up condition and less alpha in the alpha-down condition. Participants in both 
conditions were instructed to make the green bar rise as high as possible above a line, which marked their individual alpha threshold (i.e., the alpha peak-to-peak amplitude that a trainee reached or superseded $70 \%$ of the time while watching the moving bar display during a one-minute threshold setting period immediately prior to B1). To help increase their alpha brain waves, participants in the alpha-up (but not alpha-down) condition were instructed as in Fell et al.'s alpha NFB protocol to (1) relax as much as possible and breathe regularly; (2) let all thoughts, feelings, and everything else pass; and (3) let themselves sink deeper and deeper into the chair. Participants in the alpha-down NFB condition were not given instructions that were specific to that condition and that were not provided in the alpha-up NFB condition. Participants in both the alpha-up and alpha-down condition were instructed to use the rest breaks during the NFB training to look away from the screen, adjust their position, and talk to the experimenter. To limit muscle artifact, participants in both the alpha-up and alpha-down condition were presented with a feedback tone whenever the magnitude of electric myographic activity (EMG) which reflects muscle artifact in the EEG, exceeded $10 \mu \mathrm{V}$. During the baseline segments, participants watched a nonmoving version of the bar display in which the bar was kept at a constant height. Other than the NFB in between RAT1 and RAT2, participants had not received any prior training with the study's NFB protocol.

\section{Analysis of RAT performance}

The dependent variables were the number of correctly solved RAT1 and RAT2 items, the number of RAT2 items solved with or without insight, and the reaction time (RT) for correct RAT1 and RAT2 answers. RT was defined as the time elapsed in ms between the onset of the RAT problem and the space bar press, immediately prior to typing the answer. RAT performance was analyzed with by-subject ANOVAs. A one-factor ANOVA with condition (control, alpha-up, alpha-down) as a between-subjects factor examined whether participants in the three different groups were matched in terms of their RAT1 accuracy (i.e., number of solutions), RAT1 RT, and RAT2 RT. Since this turned out to be the case (all Ps $>$.45), ANOVAs of the effects of condition on RAT2 accuracy were therefore performed on the number of RAT2 solutions. A $3 \times 2 \times 2$ mixed-factor ANOVA of RAT2 solutions examined the effects of condition (control, alpha-up, alpha-down), problem difficulty (easy, hard), and solution type (insight, noninsight) with condition as a between-subjects factor and problem difficulty and solution type as within-subjects factors. Problems were divided into easy and hard problems based on a median-split by item difficulty, defined in terms of the percentage of participants who did not answer that item correctly in RAT1. Main effects of condition on RAT2 solutions were further examined using ANOVAs in terms of contrasts (i.e., contrast1: control versus incubation [i.e., averaged across alpha-up and alpha-down], contrast2: control vs. alpha-up, contrast3: control vs. alpha-down, and con-

- volume 4, no. 2 (Spring 2012) 
trast4: alpha-up vs. alpha-down). Interaction effects were broken down into simple main effects using additional ANOVAs. Only results significant at $\mathrm{p}<.05$ are reported.

\section{Analysis of EEG data}

Eye blink and other movement artifacts were removed from the EEG as described in Dien (2010). Subjects' mean, log-transformed EEG spectral power in the alpha frequency band $(8-12 \mathrm{~Hz})$ and its surrounding two frequency bands, theta $(4-8 \mathrm{~Hz})$ and beta (i.e., beta 1 and 2: $12-20 \mathrm{~Hz}$ ), were entered as data points in separate, frequency-band-specific, repeated measures ANOVA. One ANOVA crossed the factors Condition (Alpha-up, Alpha-down) and Training Segment (T1 versus T9); a second ANOVA crossed the factors Condition (Alpha-up, Alpha-down) and Baseline Segment (B1 versus B4). The comparison of T1 and T9 and B1 versus B4 to examine training effects follows the approach by Fell, et al. (2002). Effect sizes were computed as partial Eta squared values. All degrees of freedom reported are Greenhouse-Geisser corrected when applicable unless noted. As in Fell, et al. (2002), baseline segments (B) were not compared to training segments (T) (e.g. B1 to T1 and B4 to T4) since the baseline segments presented a nonchanging neurofeedback signal (e.g., nonmoving bar in our study) and the training segments a continuously changing feedback signal (moving bar in our study) and since the latter can be expected to increase alpha spectral power compared to the former, even without participants learning to change the signal due to NFB.

In addition, one-factor ANOVAs of RAT2 solutions tested the hypothesis of the presence of an association between training-related neural-electrical activity and RAT performance in the alpha band and its absence in the immediately adjacent beta and theta bands. One of these ANOVAs examined the effect of a participant grouping factor with values large alpha increase, small alpha increase, and control; a second ANOVA examined the effect of a grouping factor with values large beta increase, small beta increase, and control; and a third ANOVA examined the effect of a grouping factor with values large theta increase, small theta increase, and control. Control refers to participants in the nonNFB control condition. Large increase versus small increase in a frequency band refers to participants whose increase in spectral power in that band from training segment T1 to T9 is above or below the median, respectively, of the T1-to-T9 increase in spectral power in that band across all participants receiving NFB (i.e., across participants in both the alphaup and alpha-down NDB condition). Spectral power measured from electrode PO8 was used to define this median value, since that electrode was the basis of the neurofeedback signal. Correlations between band-specific EEG spectral power and RAT2 performance were calculated as well. Analogous ANOVAs replaced the change measure of EEG spectral activity from the start to the end of the NFB training period with a measure of the absolute alpha, beta, and theta activity during the entire NFB training period. Only results significant at $p<.05$ are reported. 


\section{Results}

\section{RAT1 performance}

Participants in the three conditions were matched in terms of their RAT1 accuracy, RAT1 RT, and RAT2 RT (all Ps>.45). They produced 17.8, 18.1, and 18.5 solutions in the alpha-up, alpha-down, and control condition, respectively (grand average 18.1 solutions).

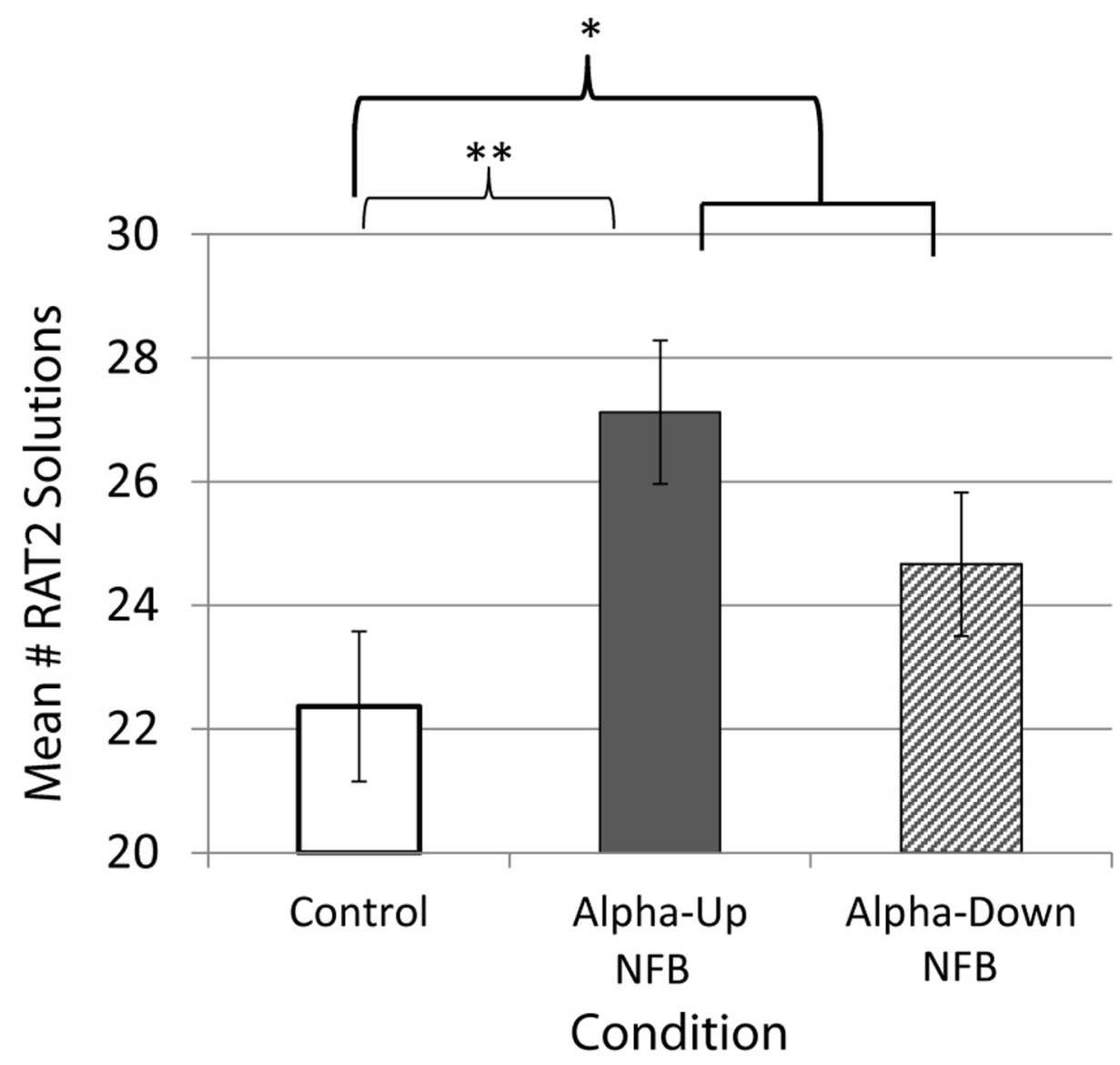

Figure 1. Mean number of RAT2 solutions as a function of condition. Error bars represent standard errors.

\section{RAT2 performance}

There was a main effect of condition (control, alpha-up, alpha-down) on number of solutions, $F(2,67)=3.881, p<.05, \eta^{2}=.104, M S E=8.112$ (see Figure 1). Contrast analysis revealed that performance was better in the incubation condition (i.e., alpha-up and alpha-down combined) than control condition, $F(1,68)=5.720, p<.05, \eta^{2}=.078$, MSE $=$ 
8.226. Furthermore, performance was better in the alpha-up than control condition $\mathrm{F}(1$, $45)=7.653, p<.01, \eta^{2}=.145, M S E=8.217$.

In addition, in the analysis of RAT2 solutions, there were main effects of solution type, $F(1,67)=71.025, p<.001, \eta^{2}=.515, M S E=18.481$, and item difficulty $F(1,67)=129.11$, $p<.001, \eta^{2}=.658, M S E=5.631$, and an interaction between solution type and difficulty $F(1,67)=15.512, p<.001, \eta^{2}=.188$, MSE $=7.589$. Solutions were reported more often with than without insight and easier items were solved more often than difficult ones. The effect of item difficulty was larger for insight, $F(1,67)=71.769, p<.001, \eta^{2}=.517$, $M S E=9.961$, than noninsight solutions, $F(2,67)=39.843, p<.001, \eta^{2}=.373, M S E=3.258$.

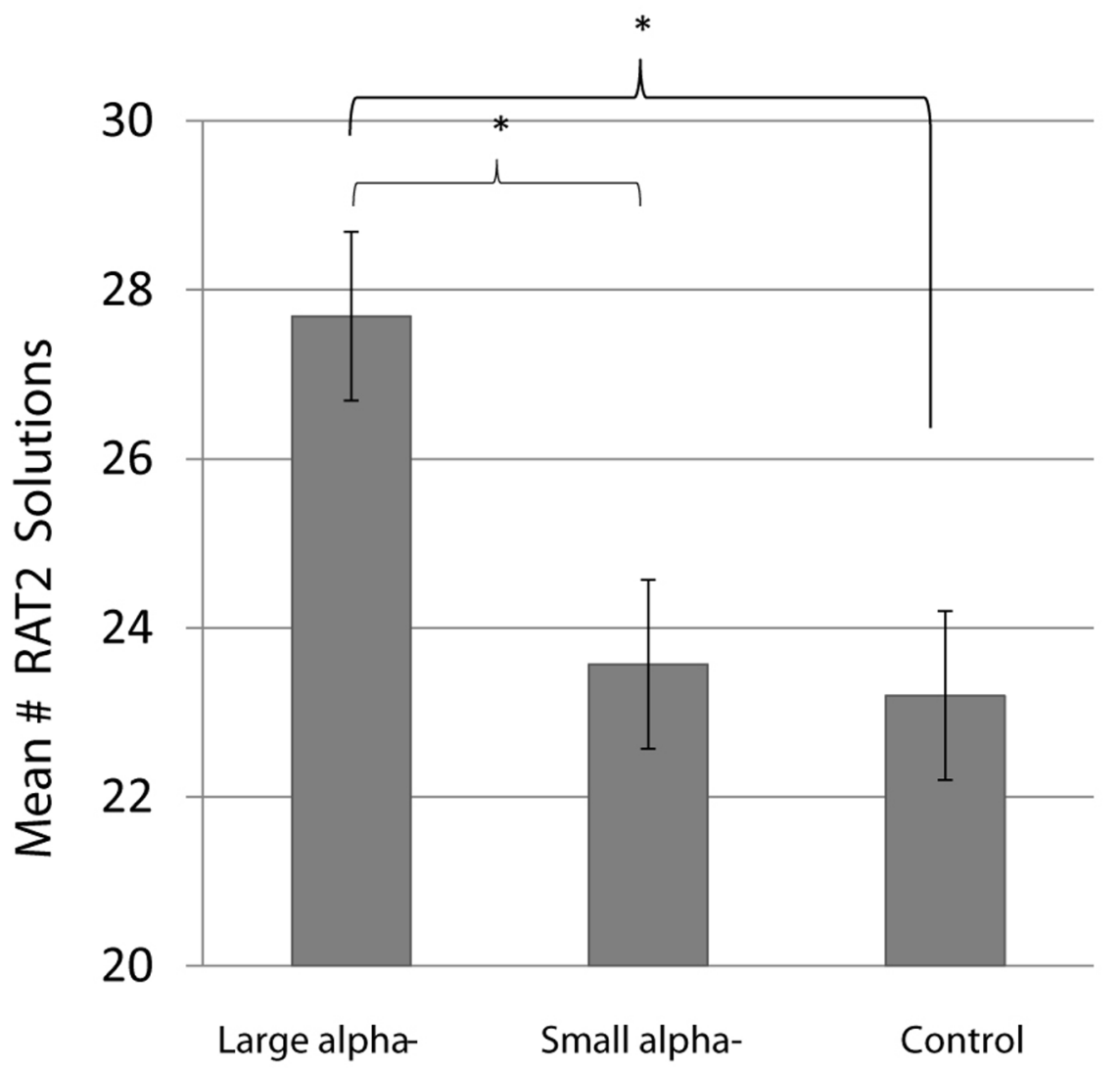

Figure 2. Mean number of RAT2 solutions in participants with a large versus small increase in alpha spectral power during neurofeedback training. Performance in the non-neurofeedback control condition is shown as well. * $p<.05$.

Finally, there was a main effect of the alpha increase factor on number of RAT2 solutions, $F(1,66)=3.688, p<.05, \eta^{2}=.101, M S E=39.121$, reflecting the greater number of RAT2 solutions in participants with a large increase in alpha spectral power during NFB compared to individuals with a small increase in alpha spectral power $(p<.05)$ and indi- 
Table 1. Number of RAT2 solutions as a function of the size of the increase in EEG spectral power and frequency band.

\begin{tabular}{llll}
\hline Band & Increase & M & SE \\
\hline \multirow{2}{*}{$\alpha$} & Large & 27.69 & 1.30 \\
& Small & 23.57 & 1.36 \\
\hline \multirow{2}{*}{$\beta$} & Large & 25.95 & 1.38 \\
& Small & 25.81 & 1.38 \\
\multirow{2}{*}{$\theta$} & Large & 25.82 & 1.34 \\
& Small & 25.86 & 1.37 \\
\hline Control & & 23.20 & 1.25 \\
\hline
\end{tabular}

viduals in the control group $(p<.05)$ (see Figure 2). By contrast, neither the degree of beta increase, $F(1,66)=1.362, p>.25, \eta^{2}=.040, M S E=42.308$, nor the degree of theta increase $(F<1, p>.95)$ predicted RAT 2 solutions (see Table 1 ).

\section{Impact of neurofeedback on brain activity}

The overall pattern of effects (see Figure 3 and Table 2) was as follows: Participants in the alpha-up and alpha-down group showed increased EEG spectral power proceeding from the first to the final baseline segment in the alpha, theta, and beta bands, and proceeding from the first to the final training segment in the theta and beta bands. Only participants in the alpha-up group showed increased EEG spectral power proceeding from the first to the final training segment in the alpha band. Furthermore, across the first and final training segments, beta power was higher in the alpha-up than alpha-down condition. Thus, in addition to EEG spectral effects that alpha-up and alpha-down NFB shared in common, EEG spectral power obtained in the alpha-up condition differed from the alpha-down condition in two ways. First, there was an increase in alpha power proceeding from the first to final training segment in the alpha-up but not alpha-down condition. Second, beta power during training was greater in the alpha-up than alpha-down condition. As described next, the ANOVAs in the different frequency bands confirmed this pattern of NFB effects on EEG spectral power. 


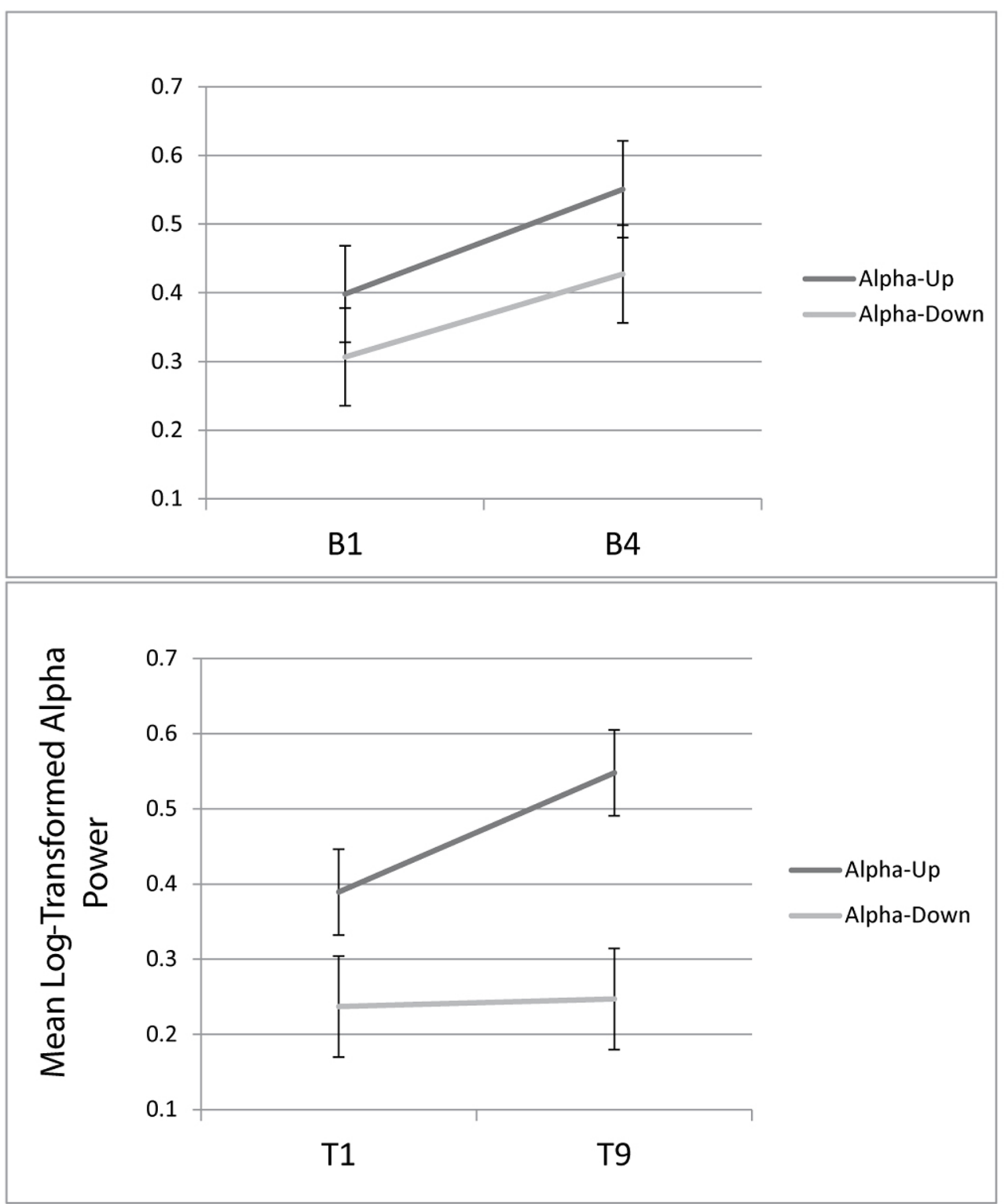

Figure 3. Mean log-transformed alpha power as a function of training segment (upper panel) and baseline segment (lower panel) in the alpha-up and alpha-down NFB condition. In order, T1 and T9 represent the first and final NFB training segment, while B1 and $B 4$ represent the first and final NFB baseline. Error bars reflect standard errors.

\section{NFB effects in alpha band}

Trainees in the alpha up and down conditions started at matched levels of alpha power at T1. Trainees in the alpha up (but not alpha down) condition increased their alpha power proceeding from T1 to T9 (see Figure $3 \&$ Table 1). This pattern of results was confirmed by the ANOVAs, which revealed main effects of training segment, $F(1,42)=15.384, p<.001$, 
Table 2. Mean EEG spectral power as a function of frequency band, NFB condition, and NFB baseline (B1 to B4) and training segment (T1 to T9).

\begin{tabular}{|c|c|c|c|c|c|c|}
\hline Band & NFB & & B1 & B2 & B3 & B4 \\
\hline \multirow{4}{*}{$\alpha$} & $\alpha-$ & $M$ & 0.370 & 0.489 & 0.506 & 0.528 \\
\hline & up & SE & 0.061 & 0.072 & 0.062 & 0.062 \\
\hline & & $M$ & 0.288 & 0.416 & 0.536 & 0.437 \\
\hline & down & SE & 0.061 & 0.062 & 0.094 & 0.065 \\
\hline \multirow{4}{*}{$\beta$} & $\alpha-$ & $M$ & 0.127 & 0.099 & 0.130 & 0.148 \\
\hline & up & SE & 0.049 & 0.057 & 0.054 & 0.059 \\
\hline & & $M$ & -0.005 & 0.086 & 0.100 & 0.045 \\
\hline & down & SE & 0.052 & 0.055 & 0.056 & 0.054 \\
\hline \multirow{4}{*}{$\theta$} & $\alpha-$ & $M$ & 0.318 & 0.337 & 0.360 & 0.357 \\
\hline & up & SE & 0.035 & 0.040 & 0.043 & 0.044 \\
\hline & & $M$ & 0.292 & 0.360 & 0.300 & 0.366 \\
\hline & & SE & 0.049 & 0.050 & 0.055 & 0.067 \\
\hline
\end{tabular}

\begin{tabular}{|c|c|c|c|c|c|c|c|c|c|c|c|}
\hline Band & NFB & & $\mathrm{T} 1$ & $\mathrm{~T} 2$ & T3 & T4 & T5 & T6 & T7 & T8 & T9 \\
\hline \multirow{4}{*}{$\alpha$} & \multirow{2}{*}{$\begin{array}{l}\alpha- \\
\text { up }\end{array}$} & $M$ & 0.389 & 0.454 & 0.519 & 0.515 & 0.494 & 0.532 & 0.487 & 0.536 & 0.548 \\
\hline & & SE & 0.057 & 0.063 & 0.071 & 0.068 & 0.060 & 0.061 & 0.062 & 0.066 & 0.067 \\
\hline & \multirow{2}{*}{$\begin{array}{l}\alpha- \\
\text { down }\end{array}$} & $M$ & 0.237 & 0.336 & 0.360 & 0.326 & 0.337 & 0.407 & 0.360 & 0.293 & 0.247 \\
\hline & & SE & 0.057 & 0.097 & 0.085 & 0.079 & 0.089 & 0.084 & 0.090 & 0.076 & 0.076 \\
\hline \multirow{4}{*}{$\beta$} & \multirow{2}{*}{$\begin{array}{l}\alpha- \\
\text { up }\end{array}$} & $M$ & 0.094 & 0.103 & 0.093 & 0.093 & 0.140 & 0.149 & 0.112 & 0.151 & 0.129 \\
\hline & & SE & 0.047 & 0.055 & 0.051 & 0.050 & 0.052 & 0.057 & 0.058 & 0.057 & 0.050 \\
\hline & \multirow{2}{*}{$\begin{array}{l}\alpha- \\
\text { down }\end{array}$} & $M$ & -0.069 & -0.001 & 0.009 & -0.028 & -0.049 & 0.059 & 0.028 & -0.026 & -0.067 \\
\hline & & SE & 0.049 & 0.064 & 0.051 & 0.051 & 0.055 & 0.055 & 0.063 & 0.048 & 0.052 \\
\hline \multirow{4}{*}{$\theta$} & \multirow{2}{*}{$\begin{array}{l}\alpha- \\
\text { up }\end{array}$} & $M$ & 0.294 & 0.315 & 0.342 & 0.313 & 0.343 & 0.359 & 0.346 & 0.379 & 0.332 \\
\hline & & SE & 0.034 & 0.040 & 0.042 & 0.039 & 0.043 & 0.043 & 0.046 & 0.046 & 0.040 \\
\hline & \multirow{2}{*}{$\begin{array}{l}\alpha- \\
\text { down }\end{array}$} & $M$ & 0.276 & 0.307 & 0.305 & 0.278 & 0.263 & 0.333 & 0.287 & 0.288 & 0.248 \\
\hline & & SE & 0.055 & 0.061 & 0.057 & 0.058 & 0.072 & 0.060 & 0.057 & 0.065 & 0.061 \\
\hline
\end{tabular}

- volume 4, no. 2 (Spring 2012) 
$\eta 2=.268, M S E=.028$, and condition $F(1,42)=4.577, p<.05, \eta 2=.098, M S E=.238$, as well as a segment $x$ condition interaction, $F(1,42)=5.551, p<.05, \eta 2=.117$, MSE $=.028$. The interaction was due to a significant increase in alpha power (a) across training segment (T1 vs. T9) in the alpha-up (but not alpha-down) condition, $F(1,24)=15.944, p<.01, \eta 2=$ $.399, M S E=.040$, and (b) at the final training segment (T9) in the alpha-up compared to the alpha-down condition, $F(1,42)=6.921, p<.05, \eta 2=.141$, MSE $=.149$. The analysis of baseline segments (B1 vs. B4) revealed a main effect of baseline segment of alpha power, $F(1,43)=20.724, p<.001, \eta 2=.325, M S E=.046$, due to an increase in alpha power across baseline segments; however, this did not interact with condition. A map of the topographic distribution of the $p$ values of the increase in alpha power during NFB training at each recording electrode revealed that the effect was most prominent over the occipital lobe and the junction of this lobe with the parietal lobe, especially for electrodes over the right hemisphere, including (but not limited to) the electrode over scalp location PO8 on which the neurofeedback signal (i.e., alpha spectral power) was based.

\section{NFB effects in theta band}

Trainees in the alpha-up and alpha-down conditions increased theta power proceeding from the first (T1) to the last (T9) training segment, $F(1,43)=12.232, p<.001, \eta 2=.221$, $M S E=.016$, and also from the first (B1) to the last (B4) baseline segments, $F(1,43)=6.037$, $p<.05, \eta 2=.123$, MSE $=.010$ (see Table 2 for means and standard errors).

\section{NFB effects in beta band}

Trainees in the alpha-up and alpha-down conditions increased beta power proceeding from the first (T1) to the last (T9) training segment, $F(1,42)=6.847, p<.05, \eta 2=.140$, MSE $=.015$, and also from the first (B1) to the last (B4) baseline segments, $F(1,43)=7.984, p<$ $.01, \eta 2=.157$, MSE = .015 (Table 2). Beta power during training (i.e., across T1 and T9) was higher in the alpha-up than alpha-down condition, $F(1,42)=5.278, p<.05, \eta 2=.112$, MSE $=.106$ (see Table 2 for means and standard errors).

\section{Discussion}

Our findings replicate and extend the RAT results obtained by Smith and colleagues (Kohn \& Smith, 2009; Smith \& Blankenship, 1991). As in their experiments, we found that RAT performance benefits from incubation under conditions of mental fixation induced by strong misleading associates presented together with the cue words. Wiley found a positive impact of incubation on RAT performance regardless of whether or not mental fixation had been induced by changing the task context (1998). Taken together, the results from these previous studies and the present study indicate that the RAT provides a useful test paradigm for studying incubation. A new result of particular interest is our finding that intentionally altering alpha brain wave activity relates to success on a problem solving 
ask. Subjects with the largest increase in alpha brain waves from the start to the end of the NFB training solved the most RAT2 problems across individuals engaged in problem incubation.

Our incubation results raise several questions of theoretical interest. Smith and colleagues obtained evidence for an incubation effect in experiments in which the incubation period came immediately after a single RAT item or after short lists of five to ten RAT items. By contrast, we obtained an incubation effect in an experiment in which the incubation period came after presenting a list with as many as 120 RAT items. Moreover, 102 RAT items were presented again in a new random order for a second solution attempt, so that on average about 111 RAT other items separated the first and second presentation of any particular RAT item. It seems quite remarkable to find an incubation effect under these conditions, since even without incubation each particular RAT item is separated by so many other RAT items that the activation levels of the misleading associates (and therefore their interfering effects) in memory are likely to have dropped considerably due to temporal decay or displacement in short-term memory. Any additional forgetting of these misleading associates during the incubation period may therefore have not been very relevant for increasing the probability of finding a RAT solution at the second problem solving attempt. The mechanism responsible for incubation under these conditions remains to be elucidated.

A second aspect of our incubation results that is of theoretical interest involves the activity during the incubation interval. In the experiments by Smith and colleagues, a RAT incubation effect was obtained when this activity involved a cognitive task (i.e., studying a story or monitoring digits), whereas in our experiment it involved EEG NFB. As mentioned above, of particular interest is our finding that a large increase in alpha activity during incubation was associated with benefitting from incubation. This finding suggests that being in an alert, calm, distraction-free (or distraction-reduced) mental state, which is reflected by increased alpha activity, is associated with benefitting from incubation or, alternatively, preparing the mind for better subsequent problem solving. Such a state might reduce processing biases that could result from effortful, top-down, explicit rulebased processing and enable better constraint satisfaction in implicit memory. A reduced contribution of the former system and increased contribution of the latter system may promote a positive impact of incubation, as assumed in the explicit implicit interaction theory (EII) of incubation effects and insight problem solving (Helie \& Sun, 2010). One might expect that the putative benefit of such an alpha-mediated processing style during incubation is more likely to emerge with greater mental fixation, as might be induced by the processing of misleading close associates. However, whether such a mechanism exists is an open empirical question, especially since in our study the problem words were always presented with such associates. Our study manipulated neither the absence versus presence of misleading associates nor the strength of their association to the problem

- volume 4, no. 2 (Spring 2012) 
words. Another open empirical question is whether improved performance associated with increased alpha activity reflects a neurocognitive mechanism linked to an incubation process or, alternatively, a process that prepares the mind for better subsequent problem solving. Our study cannot address that question, since it did not manipulate whether the alpha-up versus alpha-down NFB occurred immediately before versus after RAT1.

The above line of reasoning on the role of alpha brain waves does not exclude the possibility of a positive contribution of effortful processing to RAT performance during incubation. The finding of a positive correlation between working memory capacity and RAT performance attention (Ricks, Turley-Ames, \& Wiley, 2007) is consistent with this possibility, since it may reflect a common contribution from the executive control of attention (Ricks, et al., 2007), which involves effortful processing, for instance, in order to resolve conflict (Fernandez-Duque, Baird, \& Posner, 2000). The role of control of attention is also highlighted by the finding that high working memory capacity no longer benefits RAT performance when RAT problems are specifically constructed to activate and focus attention on strong misleading cues in memory based on a person's prior domain-specific knowledge (Ricks, et al., 2007).

The study by Jung-Beeman et al. (2004) and our study both revealed an association between alpha brain waves and RAT solutions and suggest that it may be fruitful to consider the impact of the brain and mental state that might mediate such an association. In the study by Beeman et al., insight solutions were predicted by increased alpha activity 1.4 to 0.4 seconds prior to the answer compared to noninsight solutions, while in our study individuals who showed a high increase in alpha activity during incubation benefitted from incubation whereas individuals who showed a low increase in alpha activity did not. As Jung-Beeman et al. suggested, increased alpha activity might reflect the inhibition of processing by brain regions that would otherwise activate mental representations of distracters, particularly, visual distracters processed in the occipital lobe and its junction with the parietal lobe. Such distracters would make the selection of the weak associate answer of a RAT problem even more difficult since it has to overcome competition from strong, misleading associates. In our RAT study and that by Jung-Beeman et al., the topography of the alpha increases was similar. In their study alpha increases predicting RAT performance were obtained over an electrode in a location over right parietal-occipital cortex (i.e., PO8) and in our study the alpha NFB signal and calculation of the alpha change index was based on that same electrode. Both studies show an association of RAT performance with alpha brain wave activity, using different measures. The study by Jung-Beeman et al. (2004) showed the association in terms of a short-term increase in alpha activity within a single problem and the present study revealed it in terms of an increase in tonic level of alpha activity over a broader time, across many problems.

Although the above-mentioned general mechanism may account for the association between alpha activity obtained in both our study and that by Jung-Beeman et al., 
it remains an open question to what extent the same mechanism was at work in both studies. First, alpha activity predicted insight solutions in Jung-Beeman et al.'s study but it predicted solutions regardless of whether they were produced with or without insight in our study. Second, as mentioned above, the type of alpha activity that predicted RAT performance was different in the two studies. In our study, the alpha activity that predicted RAT solutions involved an increase in the steady state EEG taking place during a thirty minute period while participants were not presented with a specific RAT problem. By contrast, in Jung-Beeman et al.'s study, it was activity that was evoked for just about a second right before participants produced an answer to a particular problem. Moreover, the relative average increase in alpha activity, which Beeman et al. found over electrode location PO8 for noninsight solutions during the 1.4 to 0.4 seconds before the answer, was superimposed on downward sloping alpha activity for both insight and noninsight solutions during that same period. This pattern of alpha activity suggests that the retrieval of the answers utilizing semantic networks in the right hemisphere was effortful but less so for insight solutions, whose retrieval by definition involve a subjective awareness of little or no effort compared to noninsight solutions.

One of the major goals of this study was to use alpha-up and alpha-down NFB to investigate the causal contribution of alpha activity during incubation to RAT performance. Unfortunately, two aspects of the results indicated that this goal remains to be achieved. First, behavioral performance in the alpha-up and alpha-down NFB condition was not reliably differentiated. While performance in the alpha-up condition was significantly better than that in the control condition, performance in the alpha-down condition was descriptively in between performance in the alpha-up and performance in the control conditions but also not significantly different from either one of them. The present study did not include a control condition that involved incubation without NFB. Adding such a condition might help to obtain an effect of alpha-up and/or alpha-down NFB on RAT solutions. However, special care would have to be taken to control for factors unrelated to the up- and down-modulation of alpha brain waves.

Second, in terms of their impact on EEG spectral power during the incubation period, the alpha-up and alpha-down NFB condition differed not only in terms of activity in the alpha band (i.e., greater increase in alpha activity from the beginning to the end of training in the alpha-up than alpha-down condition) but also in terms of activity in the beta band (i.e., greater overall beta activity in the alpha-up than alpha-down condition. Consequently, differences in RAT performance between the two conditions cannot be uniquely attributed to differences in alpha activity since there were also differences in beta activity. The increased beta activity may reflect increased effort with NFB during incubation, since participants had received no prior NFB training. It may be feasible to train participants to selectively increase their alpha brain waves with just a few NFB training sessions (Haarmann, George, Smaliy, Grunewald, \& Novick, 2009) prior to the incubation 
experiment or with other methods, such as, listening to relaxation instructions or brain stimulation with repetitive Transcranial Magnetic Stimulation (rTMS) (Klimesch, Sauseng, \& Gerloff, 2003). It might also be feasible to identify an alpha-down NFB manipulation that decreases alpha activity by avoiding the use of a moving visual feedback signal that causes continuous alpha de-synchronization (i.e., a reduction in alpha activity).

A positive result was that there was a greater number of individuals with a large increase in alpha activity in the alpha-up than alpha-down condition and, as discussed above, that across subjects in both conditions those with high alpha produced more RAT2 solutions. In view of these results, our findings involve evidence for an association of alpha brain waves with RAT performance inferred from the impact of a pseudo-experimental grouping factor (i.e., degree of change in alpha activity during NFB) but not for a causal role of these brain waves. The finding that individuals in both the alpha-up and alphadown NFB condition increased their alpha activity from the pretraining to the posttraining baseline could reflect habituation or fatigue.

As is often the case in EEG NFB, our NFB procedure used individual-specific alpha thresholds that were determined immediately before the NFB training. This raises the question of whether and how individual variation in the starting levels of alpha activity modulates the effect of NFB on alpha activity and its association with RAT performance. For example, a participant who begins at a high level of alpha activity may be lowered into the optimal alpha range with alpha-down NFB. Addressing this possibility would be an interesting direction for future work.

We hope that our approach to investigating problem solving on the RAT encourages research that determines the impact of an individual's mode of thinking on their likelihood to solve problems that require attention to weak but relevant relations (see Aiello, Jarosz, Cushen, \& Wiley, 2012; Wegbreit, Suzuki, Grabowecky, Kounios, \& Beeman, 2012, in this issue) and to benefit from incubation. It would be of considerable theoretical and practical interest to understand under what conditions and why effortful processing versus relaxed processing benefits sensitivity to weak but relevant relations as on the RAT. The findings of this study are promising in this regard, since they suggest that individuals, who show a large increase in alpha activity during incubation, increase their likelihood of solving RAT problems and since alpha activity reflects a calm and alert state in which the impact of distracters on processing is limited.

Appendix A: RAT materials

\begin{tabular}{|c|c|c|c|c|c|c|}
\hline \multicolumn{3}{|c|}{ RAT Problem } & Answer & $\begin{array}{c}\text { Strong } \\
\text { Assoc. 1 }\end{array}$ & $\begin{array}{c}\text { Strong } \\
\text { Assoc. 2 }\end{array}$ & $\begin{array}{c}\text { Strong } \\
\text { Assoc. } 3\end{array}$ \\
\hline MAIN & SWEEPER & LIGHT & STREET & man & chimney & heavy \\
\hline LIGHT & BIRTHDAY & STICK & CANDEL & feather & present & carrot \\
\hline WAY & BOARD & SLEEP & WALK & milky & water & bed \\
\hline
\end{tabular}




\begin{tabular}{|c|c|c|c|c|c|c|}
\hline DREAM & BREAK & LIGHT & $D A Y$ & sleep & hammer & bulb \\
\hline AGE & MILE & SAND & STONE & middle & distance & blast \\
\hline WHEEL & HAND & SHOPPING & CART & spoke & glove & mall \\
\hline TRIP & HOUSE & GOAL & FIELD & vacation & roof & keeper \\
\hline CHANGE & CIRCUIT & CAKE & SHORT & coin & breaker & icing \\
\hline NUCLEAR & FEUD & ALBUM & FAMILY & war & fight & record \\
\hline TOOTH & POTATO & HEART & SWEET & ache & chips & strings \\
\hline HOUND & PRESSURE & SHOT & $B L O O D$ & annoy & cooker & camera \\
\hline MARSHAL & CHILD & PIANO & GRAND & chief & adult & bar \\
\hline SENSE & COURTESY & PLACE & COMMON & feel & polite & mat \\
\hline TEETH & ARREST & START & FALSE & bite & cardiac & stop \\
\hline MAIL & BOARD & LUNG & $B L A C K$ & chain & room & breathe \\
\hline PIE & LUCK & BELLY & POT & crust & good & dancer \\
\hline POLITICAL & SURPRISE & LINE & PARTY & prisoner & shock & fishing \\
\hline RIVER & NOTE & ACCOUNT & BANK & water & melody & user \\
\hline SHINE & BEAM & STRUCK & MOON & shoe & balance & hit \\
\hline CHAMBER & MASK & NATURAL & GAS & music & costume & life \\
\hline PILE & MARKET & ROOM & STOCK & leaf & place & dining \\
\hline PINE & CRAB & SAUCE & APPLE & cone & cake & simmer \\
\hline HEALTH & TAKER & LESS & CARE & food & thief & more \\
\hline TEST & RUNNER & MAP & $R O A D$ & sheet & distance & treasure \\
\hline OFFICE & MAIL & HAT & $B O X$ & desk & stamp & straw \\
\hline FUR & RACK & TAIL & COAT & bear & magazine & cat \\
\hline BOOT & SUMMER & GROUND & CAMP & strap & hot & dirt \\
\hline TIME & BLOWN & NELSON & FULL & hour & fuse & harry \\
\hline DIVE & LIGHT & ROCKET & $S K Y$ & bar & bulb & scientist \\
\hline FOUL & GROUND & MATE & PLAY & odor & grass & first \\
\hline WAGON & BREAK & RADIO & STATION & wheel & glass & address \\
\hline BLANK & LIST & MATE & CHECK & stare & grocery & animal \\
\hline MEASURE & WORM & VIDEO & TAPE & bake & dance & camera \\
\hline SLEEPING & BEAN & TRASH & $B A G$ & dream & string & campacter \\
\hline RAIN & TEST & STOMACH & $A C I D$ & storm & score & flat \\
\hline WET & LAW & BUSINESS & SUIT & water & abide & owner \\
\hline HOME & SEA & BED & SICK & garden & shore & sleep \\
\hline LIFT & CARD & MASK & FACE & crane & greeting & Halloween \\
\hline CANE & DADDY & PLUM & SUGAR & walker & mommy & fruit \\
\hline PIKE & COAT & SIGNAL & TURN & polearm & winter & flare \\
\hline ILLNESS & BUS & COMPUTER & TERMINAL & hospital & driver & math \\
\hline
\end{tabular}

- volume 4, no. 2 (Spring 2012) 


\begin{tabular}{|c|c|c|c|c|c|c|}
\hline PEACH & ARM & TAR & PIT & cobbler & hand & sealant \\
\hline WORM & SHELF & END & BOOK & dance & cupboard & begin \\
\hline FOX & MAN & PEEP & HOLE & trot & woman & chick \\
\hline FRENCH & CAR & SHOE & HORN & fry & garage & sock \\
\hline SANDWICH & HOUSE & GOLF & CLUB & meat & home & trap \\
\hline CAT & NUMBER & PHONE & CALL & whiskers & count & book \\
\hline EIGHT & SKATE & STICK & FIGURE & nine & blade & branch \\
\hline FORCE & LINE & MAIL & AlR & lethal & queue & stamp \\
\hline FENCE & CARD & MASTER & POST & foil & birthday & slave \\
\hline FLOWER & FRIEND & SCOUT & GIRL & vase & neighbor & cub \\
\hline DUST & CEREAL & FISH & BOWL & vacuum & milk & food \\
\hline DATE & ALLEY & FOLD & BLIND & prune & bowling & blanket \\
\hline OPERA & HAND & DISH & SOAP & singer & wrist & satellite \\
\hline CROSS & RAIN & TIE & BOW & angry & cloud & knot \\
\hline FISH & MINE & RUSH & GOLD & pond & possess & hour \\
\hline TAIL & WATER & FLOOD & GATE & feather & fountain & light \\
\hline KNIFE & LIGHT & PAL & PEN & block & weight & friend \\
\hline CADET & CAPSULE & SHIP & SPACE & force & pill & parcel \\
\hline PIECE & MIND & DATING & GAME & meal & never & speed \\
\hline IRON & SHOVEL & ENGINE & STEAM & smelt & dig & motor \\
\hline PRINT & BERRY & BIRD & BLUE & news & juice & nest \\
\hline HOUSE & THUMB & PEPPER & GREEN & home & nose & shaker \\
\hline CATCHER & FOOD & HOT & $D O G$ & rye & snack & burn \\
\hline $\mathrm{HIGH}$ & DISTRICT & HOUSE & SCHOOL & mountain & vote & $\begin{array}{c}\text { improve- } \\
\text { ment }\end{array}$ \\
\hline COVER & ARM & WEAR & UNDER & up & leg & clothing \\
\hline DRESS & DIAL & FLOWER & SUN & skirt & phone & petal \\
\hline FOOD & FOWARD & BREAK & FAST & pantry & motion & dance \\
\hline MILL & TOOTH & DUST & SAW & tread & fairy & ashes \\
\hline BASKET & EIGHT & SNOW & BALL & fruit & o'clock & fresh \\
\hline FLY & CLIP & WALL & PAPER & fish & board & brick \\
\hline SAGE & PAINT & HAIR & BRUSH & wisdom & water & beard \\
\hline SAFETY & CUSHION & POINT & PIN & bike & sofa & charge \\
\hline TANK & HILL & SECRET & TOP & think & over & handshake \\
\hline MASTER & TOSS & FINGER & RING & degree & dice & nail \\
\hline RIGHT & CAT & CARBON & COPY & away & mouse & molecule \\
\hline MOUSE & BEAR & SAND & TRAP & squeak & child & blast \\
\hline MAN & GLUE & STAR & SUPER & business & stick & shooting \\
\hline
\end{tabular}




\begin{tabular}{|c|c|c|c|c|c|c|}
\hline STICK & MAKER & POINT & MATCH & poke & peace & sharp \\
\hline WATER & MINE & SHAKER & SALT & pitcher & shaft & martini \\
\hline TYPE & GHOST & SCREEN & WRITER & set & pale & silver \\
\hline ROPE & TRUCK & LINE & TOW & tie & engine & cross \\
\hline COTTAGE & SWISS & CAKE & CHEESE & lake & alps & love \\
\hline CREAM & SKATE & WATER & ICE & whipped & roller & pool \\
\hline LOSER & THROAT & SPOT & SORE & winner & neck & liver \\
\hline SHOW & LIFE & ROW & $B O A T$ & time & death & skid \\
\hline NIGHT & WRIST & STOP & WATCH & gown & ankle & go \\
\hline DUCK & FOLD & DOLLAR & $B I L L$ & sitting & laundry & American \\
\hline ROCKING & WHELEL & $\mathrm{HIGH}$ & CHAIR & horse & car & low \\
\hline DEW & COMB & BEE & HONEY & mountain & hair & spelling \\
\hline FOUNTAIN & BAKING & POP & SODA & park & apron & bang \\
\hline PRESERVE & RANGER & TROPICAL & FOREST & Strawberry & rescue & flavor \\
\hline AID & RUBBER & WAGON & BAND & $\begin{array}{c}\text { Humani- } \\
\text { tarian }\end{array}$ & glue & covered \\
\hline FLAKE & MOBILE & CONE & SNOW & corn & phone & traffic \\
\hline CRACKER & FLY & FIGHTER & FIRE & jack & plane & prize \\
\hline SPOON & CLOTH & CARD & TABLE & fork & fabric & playing \\
\hline CUT & CREAM & WAR & COLD & board & sour & peace \\
\hline NOTE & CHAIN & MASTER & $K E Y$ & taker & wallet & $\begin{array}{c}\text { command- } \\
\text { er }\end{array}$ \\
\hline SHOCK & SHAVE & TASTE & AFTER & electric & close & aesthetic \\
\hline GRASS & KING & MEAT & CRAB & lawn & burger & beef \\
\hline BREAK & BEAN & CAKE & COFFEE & smash & refried & clump \\
\hline CRY & FRONT & SHIP & BATTLE & scream & rear & postage \\
\hline ROLL & BEAN & $\mathrm{FISH}$ & JELLY & tuck & string & barrel \\
\hline HORSE & HUMAN & DRAG & RACE & around & being & net \\
\hline BOTTOM & CURVE & $\mathrm{HOP}$ & $B E L L$ & drawer & smooth & sock \\
\hline TOMATO & BOMP & PICKER & CHERRY & garden & shell & upper \\
\hline PEA & SHELL & CHEST & NUT & pod & half & drawer \\
\hline LINE & FRUIT & DRINK & PUNCH & queue & basket & eat \\
\hline BUMP & EGG & STEP & GOOSE & night & scrambled & kick \\
\hline FIGHT & CONTROL & MACHINE & GUN & knife & panel & tool \\
\hline HOME & ARM & ROOM & REST & garden & leg & living \\
\hline CHILD & SCAN & WASH & BRAIN & support & barcode & car \\
\hline NOSE & STONE & BEAR & BROWN & job & wall & arms \\
\hline CONTROL & PLACE & RATE & BIRTH & remote & setting & crime \\
\hline
\end{tabular}

- volume 4, no. 2 (Spring 2012) 


\begin{tabular}{|c|c|c|c|c|c|c|}
\hline LOUNGE & HOUR & NAPKIN & COCKTAIL & music & minute & silverware \\
\hline ARTIST & HATCH & ROUTE & ESCAPE & paint & bird & highway \\
\hline PET & BOTTOM & GARDEN & ROCK & teacher & barrel & salad \\
\hline MATE & SHOES & TOTAL & RUNNING & bed & heels & cereal \\
\hline SELF & ATTORNEY & SPENDING & DEFENSE & reflect & case & grocery \\
\hline LAND & HAND & HOUSE & FARM & sea & cards & doctor \\
\hline SHADOW & CHART & DROP & EYE & boxer & flow & raise \\
\hline BACK & STEP & SCREEN & DOOR & bone & hop & survey \\
\hline READING & SERVICE & STICK & LIP & gist & business & walking \\
\hline OVER & PLANT & HORSE & POWER & cross & leaf & saddle \\
\hline
\end{tabular}

\section{References}

Aiello, D. A., Jarosz, A. F., Cushen, P. J., \& Wiley, J. (2012). Firing the executive: When an analytic approach to problem solving helps and hurts. The Journal of Problem Solving, 4(2), Article 6.

Beeman, M. J., Bowden, E. M., Haberman, J., Frymiare, J. L., Arambel-Liu, S., Greenblatt, R., et al. (2004). Neural activity when people solve verbal problems with insight. PLoS Biol, 2(4), E97.

Bowden, E. M., \& Beeman, M. J. (1998). Getting the right idea: Semantic activation in the right hemisphere may help solve insight problems. Psychological Science, 9, 435-440.

Bowden, E. M., \& Beeman, M. J. (2000). The right hemisphere maintains solution-related activation for yet-to-be-solved problems. Memory \& Cognition, 28(7), 1231-1241.

Bowden, E. M., \& Beeman, M. J. (2003a). Aha! Insight experience correlates with solution activation in the right hemisphere. Psychonomic Bulletin \& Review, 10(3), 730-737.

Bowden, E. M., \& Beeman, M. J. (2003b). Normative data for 144 compound remote associate problems. Behav Res Methods Instrum Comput, 35(4), 634-639.

Bowden, E. M., \& Jung-Beeman, M. (2003). Normative data for 144 compound remote associate problems. Behav Res Methods Instrum Comput, 35(4), 634-639.

Browne, B. A., \& Cruse, D. F. (1988). The Incubation Effect: Illusion or Illumination? Human Performance, 1(3), 177-185.

Cooper, N. R., Croft, R. J., Dominey, S. J., Burgess, A. P., \& Gruzelier, J. H. (2003). Paradox lost? Exploring the role of alpha oscillations during externally vs. internally directed attention and the implications for idling and inhibition hypotheses. Int J Psychophysiol, 47(1), 65-74.

Danko, S., Shemyakina, N. V., Nagornova, Z. V., \& Starchenko, M. (2009). Comparison of the effects of the subjective complexity and verbal creativity on the EEG spectral power parameters. Human Physiology, 35, 3. 
Dietrich, A., \& Kanso, R. (2010). A review of EEG, ERP and Neuroimaging Studies of Creativity and Insight. Psychological Bulletin, 136(5), 17.

Dorfman, J., Kihlstrom, J. F., \& Shames, V. A. (1996). Intuition, Incubation, and Insight: implicit cognition and problem solving. In G. Underwood (Ed.), Implicit cognition. Oxford: Oxford University Press.

Fell, J., Elfadil, H., Klaver, P., Roschke, J., Elger, C. E., \& Fernandez, G. (2002). Covariation of spectral and nonlinear EEG measures with alpha biofeedback. International Journal of Neuroscience, 112(9), 1047.

Fernandez-Duque, D., Baird, J. A., \& Posner, M. I. (2000). Executive attention and metacognitive regulation. Consciousness and Cognition, 9, 288-307.

Fink, A., Grabner, R. H., Benedek, M., \& Neubauer, A. C. (2006). Divergent thinking training is related to frontal electroencephalogram alpha synchronization. European Journal of Neuroscience, 23(8), 2241-2246.

Fink, A., \& Neubauer, A. C. (2006). EEG alpha oscillations during the performance of verbal creativity tasks: Differential effects of sex and verbal intelligence. International Journal of Psychophysiology, 62(1), 46-53.

Haarmann, H. J., \& Cameron, K. A. (2005). Active maintenance of sentence meaning in working memory: Evidence from EEG coherences. International Journal of Psychophysiology, 57, 115-128.

Haarmann, H. J., George, T., Smaliy, A., Grunewald, K., \& Novick, J. (2009). Alpha neurofeedback training and its implications for studies of cognitive creativity. Paper presented at the 16th Annual Meeting of the Cognitive Neuroscience Society, San Francisco, CA, March 21-24.

HansImayr, S., Sauseng, P., Doppelmayr, M., Schabus, M., \& Klimesch, W. (2005). Increasing individual upper alpha power by neurofeedback improves cognitive performance in human subjects. Appl Psychophysiol Biofeedback, 30(1), 1-10.

Helie, S., \& Sun, R. (2010). Incubation, insight, and creative problem solving: a unified theory and a connectionist model. Psychological Review, 117(3), 994-1024.

Jausovec, N. (2000). Differences in Cognitive Processes between Gifted, Intelligent, Creative, and Average Individuals While Solving Complex Problems: An EEG Study. Intelligence, 28(3), 213-237.

Jensen, O., Gelfand, J., Kounios, J., \& Lisman, J. E. (2002). Oscillations in the alpha band $(9-12 \mathrm{~Hz})$ increase with memory load during retention in a short-term memory task. Cerebral Cortex, 877-882.

Jung-Beeman, M. (2005). Bilateral brain processes for comprehending natural language. Trends in Cognitive Sciences, 9(11), 512-518.

Jung-Beeman, M., Bowden, E. M., Haberman, J., Frymiare, J. L., Arambel-Liu, S., Greenblatt, R., et al. (2004). Neural activity when people solve verbal problems with insight. PLoS Biol, 2(4), E97.

- volume 4, no. 2 (Spring 2012) 
Klimesch, W. (1999). EEG alpha and theta oscillations reflect cognitive and memory performance: a review and analysis. Brain Research Reviews, 29(2-3), 169-195.

Klimesch, W., Doppelmayr, M., Rohm, D., Pollhuber, D., \& Stadler, W. (2000). Simultaneous desynchronization and synchronization of different alpha responses in the human electroencephalograph: a neglected paradox? Neurosci Lett., 284(1-2), 97-100.

Klimesch, W., Sauseng, P., \& Gerloff, C. (2003). Enhancing cognitive performance with repetitive transcranial magnetic stimulation at human individual alpha frequency. European Journal of Neuroscience, 17(5), 1129-1133.

Kohn, N., \& Smith, S. M. (2009). Partly versus completely out of your mind: effects of incubation and distraction on resolving fixation. Journal of Creative Behavior, 43(2), 102-118.

Leins, U., Goth, G., Hinterberger, T., Klinger, C., Rumpf, N., \& Strehl, U. (2007). Neurofeedback for children with ADHD: a comparison of SCP and Theta/Beta protocols. Applied Psychophysiological Biofeedback, 32(2), 73-88.

Martindale, C., \& Hasenfus, N. (1978). EEG differences as a function of creativity, stage of the creative process, and effort to be original. Biol Psychol, 6(3), 157-167.

Martindale, C., \& Hines, D. (1975). Creativity and cortical activation during creative, intellectual and eeg feedback tasks. Biological Psychology, 3(2), 91-100.

Mednick, S. A. (1962). The associative basis of the creative process. Psychological Review, 69, 220-232.

Mednick, S. A. (1968). The Remote Associates Test. Journal of Creative Behavior, 2(3), 213-214.

Mednick, S. A., \& Mednick, M. T. (1967). Remote associates test examiner's manual. Boston: Houghton Mifflin.

Molle, M., Marshall, L., Wolf, B., Fehm, H. L., \& Born, J. (1999). EEG complexity and performance measures of creative thinking. Psychophysiology, 36(1), 95-104.

Ricks, T. R., Turley-Ames, K. J., \& Wiley, J. (2007). Effects of working memory capacity on mental set due to domain knowledge. Memory \& Cognition, 35(6), 1456-1462.

Sabate, M., Llanos, C., Enriquez, E., Gonzalez, B., \& Rodriguez, M. (2011). Fast modulation of alpha activity during visual processing and motor control. Neuroscience, 189, 236-249.

Shemyakina, N. V., \& Danko, S. (2007). Changes in the power and coherence of the $\beta 2$ EEG band in subjects performing creative tasks using emotionally significant and emotionally neutral words. Human Physiology, 33, 7.

Smith, S. M., \& Blankenship, S. E. (1989). Incubation effects. Bulletin of the Psychonomic Society, 27, 311-3114.

Smith, S. M., \& Blankenship, S. E. (1991). Incubation and the Persistence of Fixation in Problem Solving. The American Journal of Psychology, 104(1), 61-87.

Sterman, M. B., \& Egner, T. (2006). Foundation and practice of neurofeedback for the treatment of epilepsy. Appl Psychophysiol Biofeedback, 31(1), 21-35.

Thut, G., Nietzel, A., Brandt, S. A., \& Pascual-Leone, A. (2006). Alpha-band electroencephalographic activity over occipital cortex indexes visu- ospatial attention bias and predicts visual target detection. Journal of Neuroscience(26), 9494-9502. 
Vernon, D. J. (2005). Can Neurofeedback Training Enhance Performance? An Evaluation of the Evidence with Implications for Future Research. Applied Psychophysiology \& Biofeedback, 30(4), 347-364.

Vernon, D. J., Egner, T., Cooper, N., Compton, T., Neilands, C., Sheri, A., et al. (2003). The effect of training distinct neurofeedback protocols on aspects of cognitive performance. International Journal of Psychophysiology, 47(1), 75-85.

Vul, E., \& Pashler, H. (2007). Incubation benefits only after people have been misdirected. Memory \& Cognition, 35(4), 701-710.

Wegbreit, E., Suzuki, S., Grabowecky, M., Kounios, J., \& Beeman, M. (2012). Visual attention modulates insight versus analytic solving of verbal problems. The Journal of Problem Solving, 4(2), Article 5.

Wiley, J. (1998). Expertise as mental set: The effects of domain knowledge in creative problem solving. Memory \& Cognition, 26(4), 716-730.

\section{Acknowledgments}

This research is based upon work supported, in whole or in part, with funding from the United States Government. Any opinions, findings and conclusions, or recommendations expressed in this material are those of the author(s) and do not necessarily reflect the views of the University of Maryland, College Park and/or any agency or entity of the United States Government. Nothing in this article is intended to be and shall not be treated or construed as an endorsement or recommendation by the University of Maryland, United States Government, or the authors of the product, process, or service that is the subject of this article. We are grateful to David Vernon, Marius Usher, and Eddy Davelaar for sharing their proposal to improve RAT performance through alpha neurofeedback and to David Vernon for suggestions for analysis of the EEG data from the NFB protocol. We thank Jared Novick for comments on this research. We also thank Suzanne Freynik and Jeffrey Chrabaszcz for helping collect data. Parts of this work have been presented as a conference poster (Haarmann, et al., 2010). Correspondence concerning this article should be addressed to Henk J. Haarmann, Center for Advanced Study of Language, University of Maryland, 7005 52nd Avenue, College Park, MD 20742 (e-mail: hhaarmann@casl.umd.edu). 